\title{
Microwave reflections from a vacuum ultraviolet laser produced plasma sheet
}

\author{
K. L. Kelly, J. E. Scharer, ${ }^{\text {a) }}$ G. Ding, M. Bettenhausen, and S. P. Kuo ${ }^{\text {b) }}$ \\ Department of Electrical and Computer Engineering, 1500 Engineering Drive, \\ University of Wisconsin - Madison, Madison, Wisconsin 53706
}

(Received 11 May 1998; accepted for publication 2 October 1998)

\begin{abstract}
A pulsed, $193 \mathrm{~nm}$ excimer laser is utilized to photoionize the organic gas tetrakisdimethylamino-ethylene (TMAE). The laser ionizes a plasma sheet with a width of $7.8 \mathrm{~cm}$ and an adjustable thickness of $0.7-1.4 \mathrm{~cm}$. The axial scale length of the plasma density is a function of TMAE neutral pressure and is typically $50 \mathrm{~cm} . X$-band $(10 \mathrm{GHz})$ microwaves are incident on the plasma with the electric field polarized parallel to the laser beam axis. The power reflection coefficient and the phase of the reflected signal are studied as a function of time. A monostatic homodyne detection system with a response time of $10 \mathrm{~ns}$ is utilized to determine the amplitude and phase of the reflected wave. The peak plasma density is $n_{e} \approx 4 \times 10^{13} \mathrm{~cm}^{-3}$, sufficiently above the critical density $\left(n_{\text {crit }}=1.2 \times 10^{12} \mathrm{~cm}^{-3}\right.$ ) to produce reflections comparable to a conducting sheet placed in the same position as the plasma. A computer model is developed to interpret and optimize the plasma conditions which provide the highest backscatter and phase-stable reflection coefficient for the longest period of time. The presence of axial density gradients causes the reflected wave to be scattered through a wide angle. As the gradients relax, the backscatter reflection coefficient increases to a value of nearly $100 \%$. The plasma density and two-body recombination coefficient are measured by means of microwave backscatter plasma reflectivity and Langmuir probes. (C) 1999 American Institute of Physics. [S0021-8979(99)08501-1]
\end{abstract}

\section{INTRODUCTION}

There have been several studies ${ }^{1-4}$ of a plasma as a microwave reflector or absorber based on its application in the field of radar communications. The primary interest is in using the plasma as an agile mirror, an alternative to a complex phased-array radar system for radar beam steering purposes. The agile mirror is attractive because of its short turn on/off time, high reflectivity, and because it has negligible inertia compared to mechanical reflectors. Studies at the Naval Research Laboratory ${ }^{1}$ illustrate that a plasma sheet generated by means of a linear hollow cathode discharge yields a far-field antenna pattern very close to that from a metal plate. The optically generated plasma in this experiment has a high plasma density making it useful for the reflection of higher frequency microwaves and has a turn-on time of $\tau_{\mathrm{on}}=10 \mathrm{~ns}$. Previous experiments by our research group 5 have also made detailed measurements of microwave absorption, transmission and backscatter from a test dipole in an electron cyclotron heating $(\mathrm{ECH})$ produced plasma in the 2-4 GHz microwave frequency range.

This experiment consists of launching $X$-band microwaves from a directive horn antenna and studying their interaction with the plasma. The reflectivity can be described by the plasma dielectric constant ${ }^{6}$

$$
\epsilon_{p}=\epsilon\left[1-\frac{\omega_{p}^{2}}{\omega(\omega+i \nu)}\right],
$$

${ }^{a}$ Electronic mail: scharer@cptc.wisc.edu

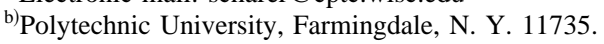

where $\omega_{p}^{2}=\left(n e^{2}\right) /(m \epsilon)$ is the plasma frequency, $m$ is the mass of the electron, $\epsilon$ is the permittivity of free space, $\omega$ is the microwave operating frequency, and $\nu$ is the electronneutral momentum collision frequency. The normalized wave impedence of such a plasma is given by

$$
\eta_{p}=\sqrt{\frac{\epsilon}{\epsilon_{p}}}=\left[1-\frac{\omega_{p}^{2}}{\omega(\omega+i \nu)}\right]^{-1 / 2} .
$$

The reflection coefficient for a normal incidence wave at a dielectric boundary is given by the Fresnel reflection coefficient,

$$
\Gamma=\frac{E_{\text {reflected }}}{E_{\text {incident }}}=\frac{\eta_{p}-1}{\eta_{p}+1} .
$$

This article carries out microwave measurements and computer simulations on a laser produced plasma with density profiles similar to that previously measured by Shen et al. ${ }^{7}$

\section{LAB APPARATUS}

Figure 1 shows the apparatus used in the experiment. An excimer laser (Lumonics Pulsemaster PM-842) produces a $193 \mathrm{~nm}$ vacuum ultraviolet (VUV) beam. The $\left(\frac{1}{2} \max \right)$ pulse width is $20( \pm 2)$ ns with a maximum laser energy of $300 \mathrm{~mJ}$. The laser output cross section is $3.0 \mathrm{~cm} \times 1.4 \mathrm{~cm}$. A customdesigned fused silica (Suprasil) VUV-coated (98\% transmission) cylindrical lens system is used to modify the cross section of the beam in the $\hat{x}$ dimension into a sheet beam measuring $7.8 \mathrm{~cm} \times 1.4 \mathrm{~cm}$. The beam also passes through an 


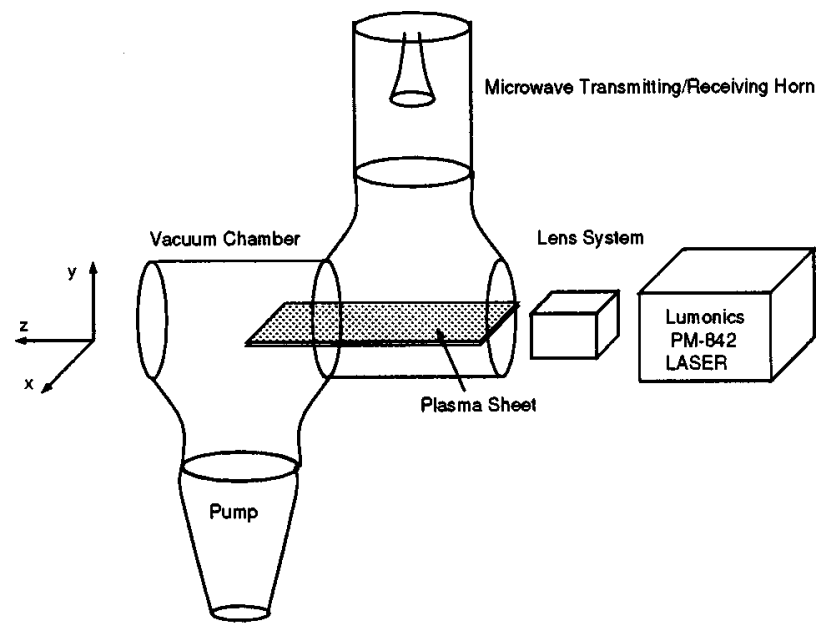

FIG. 1. Schematic of the laser produced plasma experiment.

opaque mask which provides clearly defined boundaries in the $\hat{x}$ and $\hat{y}$ directions. The lens system is shown in Fig. 2.

After the laser beam has been modified by the lenses and mask system, the intensity is measured using a fast VUV photodiode (model S 1226-18 Bz). The photodiode measurements show that the beam is most intense and flat $(\Delta I / I$ $<10 \%$ ) over a $7.8 \mathrm{~cm} \times 0.7 \mathrm{~cm}$ area.

The plasma chamber is a combination of two 6-in.-diam Corning glass Tees with three openings. The total chamber length is $100 \mathrm{~cm}$ but the region of the plasma where microwaves are incident extends $50 \mathrm{~cm}$ from the laser input window. The laser beam enters the chamber through a $9.5 \mathrm{~cm}$ window mounted over one end Tee opening. The window is coated for $98 \%$ transparency at $193 \mathrm{~nm}$. Diagnostic Langmuir probes, a photodiode, and tetrakis-dimethylaminoethylene (TMAE) gas flow valves are located at the opposite end of the chamber.

The microwave source is a (HP 8690B) sweep oscillator with an output power of $60 \mathrm{~mW}$. The source is tunable over the entire $X$-band $(8.2-12.4 \mathrm{GHz})$ range. A monostatic homodyne detection system is used to determine the amplitude and phase of the reflected signal. The detectors are silicon

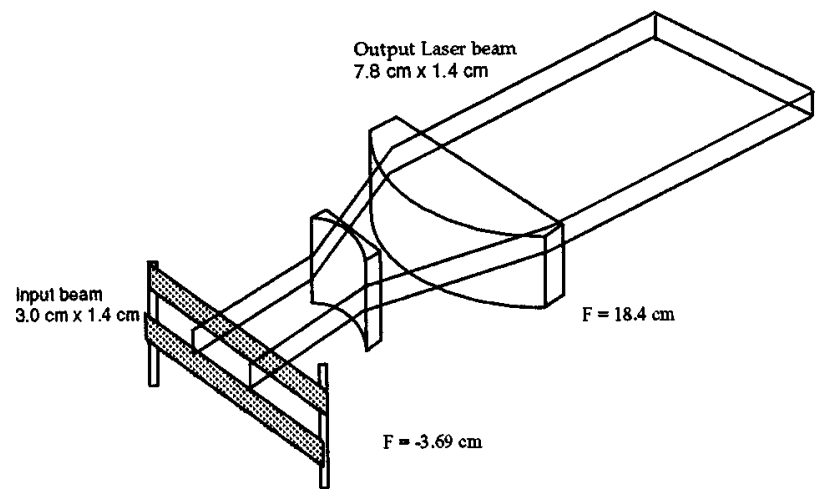

FIG. 2. Cylindrical fused silica lens system for expanding laser beam and forming plasma sheet. point contact detector diodes mounted in (HP X485B) tunable waveguide mounts. The mounts are tuned for maximum sensitivity. A calibration is performed on each detector to determine its current-voltage $(I-V)$ curve. The power received at the backscatter diode with no plasma present provides a measure of the cross section of the experimental apparatus that can be used to determine the error in measuring the plasma reflectivity. A frequency is chosen such that internal reflections from the glass chamber and waveguide system are a minimum $(\leqslant 60 \mu \mathrm{W})$. With this reflected power at a minimum, the frequency is $10.005 \mathrm{GHz}$ as measured by a cavity resonator in the microwave circuit. At the conclusion of the experiment, an aluminum sheet is inserted into the chamber and aligned with the front edge of the laser beam $( \pm 0.1 \mathrm{~mm})$. This aluminum sheet serves as a reference for a reflection coefficient of $R=1$ and a phase shift of $\phi=\pi$ radians. The aluminum sheet has a radar cross section comparable to its physical size of $A=240 \mathrm{~cm}^{2}$. The power detected at the receiver is $3.15 \mathrm{~mW}$. The given parameters predict ${ }^{8}$ that with $50 \mathrm{~mW}$ of radiated power, $3 \mathrm{~mW}$ will be detected by the receiving antenna. It is found that this falls within $5 \%$, an acceptable value considering the error in measuring distances, gain and the antenna pattern for the system. Based on these measurements, we can infer a maximum signal from the plasma of 50 times greater than the signal from the glass.

The transmitting and receiving antenna is a tapered circular waveguide horn with an aperture diameter of $d$ $=7.0 \mathrm{~cm}$ and a beamwidth of $\psi=28_{\text {FWHM }}^{0}$. Measurements are done with the transmitted wave polarized with the electric field parallel to the $\hat{z}$ axis. Radiation-field distance ${ }^{8}$ is 30 $\mathrm{cm}$ for accurate measurement of radar cross sections. A thin mylar membrane is used to maintain vacuum at the interface of the waveguide system and vacuum chamber. The detection system and calibration were checked by measuring the reflection coefficient of a sample of polystyrene with known thickness and dielectric constant. The measurements were within $10 \%$ of the known values for this sample.

Data are collected using a Tektronix 350 TDS 2 channel digitizing oscilloscope (maximium sampling frequency 1 Gs/s) and transferred to an IBM PC using National Instruments LabView interface software.

\section{PLASMA CONDITIONS}

The plasma is formed in $10 \mathrm{~ns}$ by a VUV laser operating in the $193 \mathrm{~nm}(6.42 \mathrm{eV})$ argon-flouride mode. The vertical ionization energy for TMAE is $6.11( \pm 0.02) \mathrm{eV} .{ }^{9}$ The laser has an internal feedback which adjusts cathode voltages and halogen concentration which limits the shot-to-shot power variation to $\Delta E / E<5 \%$. The laser ionizes a small fraction of the available TMAE atoms and also produces excited states of TMAE which may give rise to further ionization of the remaining TMAE molecules after the laser is extinguished. A Langmuir probe is used to monitor the plasma density and is located $50 \mathrm{~cm}$ from the laser window. The $1 / e$ photon absorption length, $\alpha_{r}$, at 60 mTorr is calculated to be $48 \mathrm{~cm} .{ }^{4}$ The initial plasma density near the laser input window is of the order $n_{e} \approx 4 \times 10^{13} \mathrm{~cm}^{-3}$ and $T_{e}=0.3 \mathrm{eV}$ as measured by the Langmuir probes as shown in Fig. 3. The 


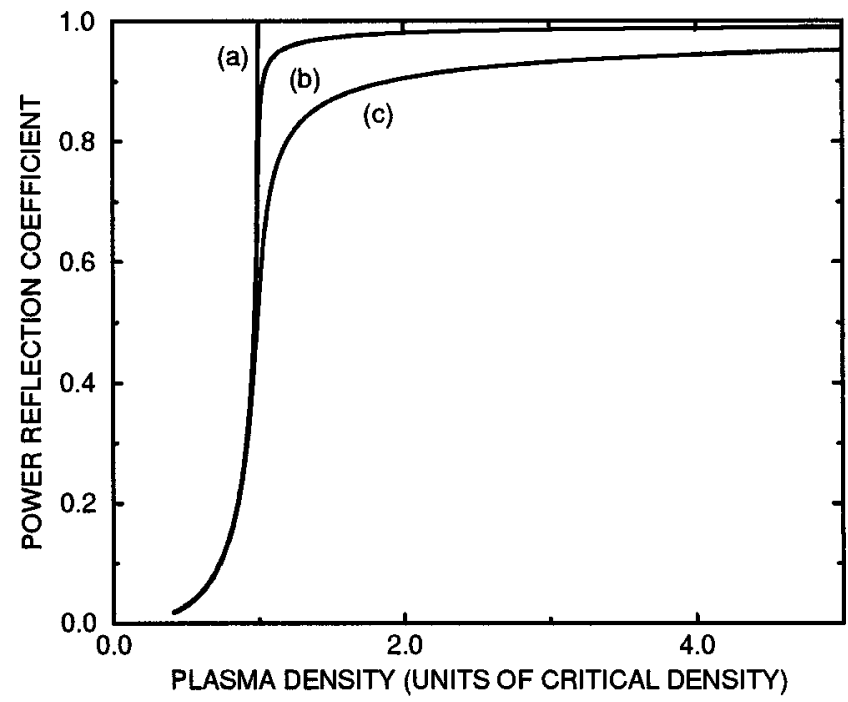

FIG. 3. Normalized electron density for a plasma by use of a Langmuir probe positioned $50 \mathrm{~cm}$ from the laser window.

lifetime of the excited states is short compared to the plasma lifetime and within 150-200 ns the plasma is in a source-free decaying state. The plasma decay is then primarily determined by two-body recombination. ${ }^{7}$ With the assumption that the number of electrons and the number of ions are equal $\left(n_{e}=n_{i}\right)$, the decay is proportional to $n_{e}$ and $n_{i}$ and can be represented by the differential equation

$$
\frac{\partial n_{e}}{\partial t}=-\alpha n_{e}^{2}
$$

where $n_{e}$ is the plasma electron density and $\alpha$ is the plasma two-body recombination coefficient. Equation (4) is solved to give

$$
n_{e}=\frac{n_{0}}{1+n_{0} \alpha t} .
$$

Previous measurements of the recombination rate ${ }^{4}$ of TMAE in atmospheric pressure helium yield a value of $\alpha=9.0$ $( \pm 1.1) \times 10^{-6} \mathrm{~cm}^{3} \mathrm{~s}^{-1}$ for $T_{e}=300 \mathrm{~K}$ when the plasma was created by means of spark gaps.

The electron-neutral collision term at 60 mTorr is negligible $(\nu<0.01 \omega) .{ }^{10}$ Our calculations show that this magnitude does not contribute to significant microwave power absorption. This can be seen from Fig. 4 which shows the theoretical power reflection coefficient at a single dielectric interface for an infinitely thick plasma sheet as described by Eq. (3). A small amount of microwave radiation may penetrate into the plasma while the plasma density is near cutoff, but these contributions are negligible considering the plasma density is within $10 \%$ of critical density for about $5 \mathrm{~ns}$, for typical recombination coefficients $\left(\alpha \sim 10^{-6} \mathrm{~cm}^{3} \mathrm{~s}^{-1}\right)$ for TMAE.

As the plasma falls below the critical density, it quickly loses its reflective properties. For example, a plasma with density $n_{e}=0.9 n_{\text {crit }}$ yields a power reflection coefficient of about $25 \%$.

We have previously shown that the initial density decay time $(t \leqslant 100 \mathrm{~ns})$ is short in comparison to the time scale for

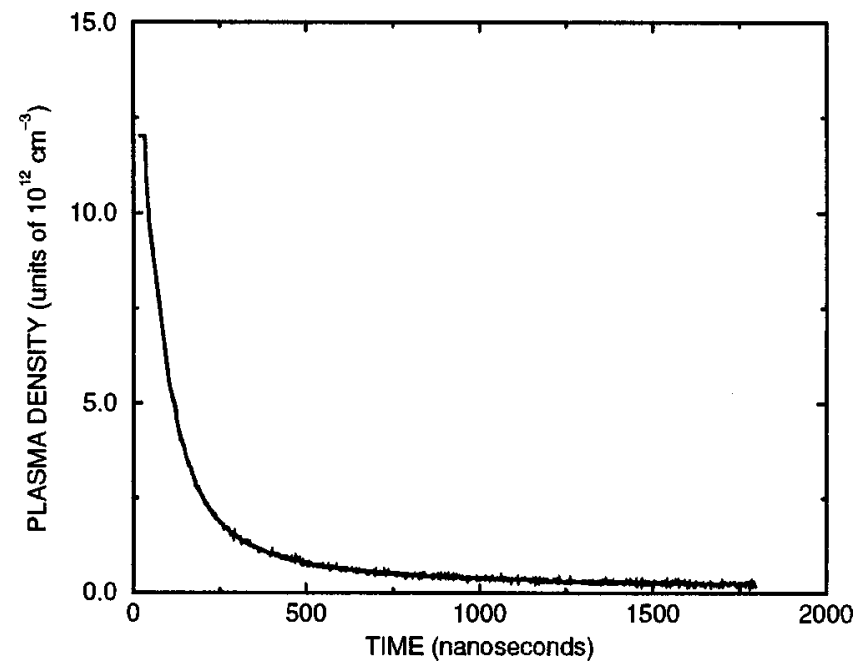

FIG. 4. Theoretical calculation of power reflection coefficient from a semiinfinite low-collisionality plasma sheet (a) noncollisional, (b) $\nu / \omega=0.01$, and (c) $\nu / \omega=0.05$.

which the plasma diffuses. ${ }^{7}$ The ambipolar diffusion coefficient was found to be $D_{a} \approx 1.1 \times 10^{4} \mathrm{~cm}^{2} \mathrm{~s}^{-1}$. Only a small number $\left(n_{e} \ll 10^{12} \mathrm{~cm}^{-3}\right)$ of electrons diffuse an appreciable fraction of a wavelength $(d=0.01 \lambda)$ before they recombine. For this reason, the boundary of the plasma remains relatively sharp in comparison to a microwave wavelength over the lifetime of the experiment.

The initial plasma density is a function of the spatial coordinates $\hat{x}, \hat{y}$, and $\hat{z}$. The $\hat{x}$ dependence is governed by the measured laser intensity profile in that dimension with a Gaussian beam assumed. Langmuir probe and photodiode measurements show that the density variation at $t=100 \mathrm{~ns}$ from the center of the beam to the edge $(x=3.8 \mathrm{~cm})$ is $20 \%$. The $\hat{y}$ density dependence is a $0.7-1.4 \mathrm{~cm}$ width step profile with sharp boundaries $(\Delta y \ll \lambda)$. The $\hat{z}$ profile is governed by the mechanism of photon absorption and plasma recombination. The amount of laser radiation available for photoionization at position $z$ is proportional to the number of photons absorbed upstream $\left(z_{0}<z\right)$. This initial condition predicts an exponential profile in the $\hat{z}$ direction. These considerations are important in that the phase of reflected signals is a function of plasma density and diffraction will result from the systematic density gradients. This results in a scattered field pattern which differs greatly from the pattern of the antenna.

\section{EXPERIMENTAL PROCEDURE AND RESULTS}

A mechanical rotary vane pump and high-vacuum diffusion pump are used to evacuate the plasma chamber. The chamber is evacuated to $P=5 \times 10^{-5}$ Torr to ensure gas purity. The vapor pressure of TMAE is $P_{\text {vapor }}=740 \mathrm{mTorr}$ at $25^{\circ} \mathrm{C} .{ }^{11}$ The needle valve is opened and a small amount of TMAE vaporizes to raise the neutral pressure to $10-150$ mTorr. The evacuate and refill procedure is repeated several times throughout the experiment to minimize impurities. 


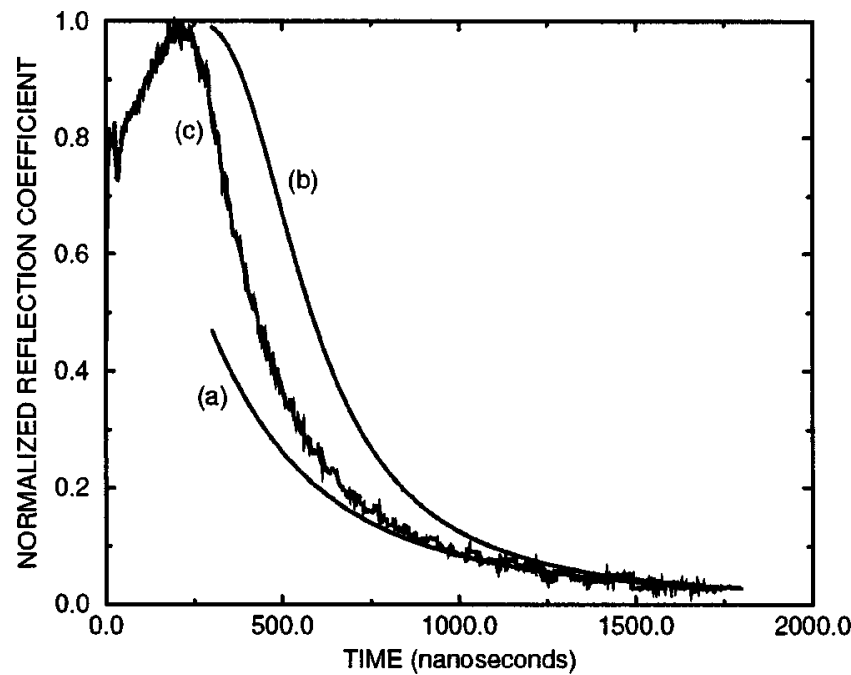

FIG. 5. Power reflection coefficient vs time for a $0.7 \mathrm{~cm}$ thick plasma (a) as simulated with a recombination coefficient $\alpha=2.0 \times 10^{-6} \mathrm{~cm}^{3} \mathrm{~s}^{-1}$, (b) $\alpha$ $=2.5 \times 10^{-6} \mathrm{~cm}^{3} \mathrm{~s}^{-1}$, (c) determined by actual measurement.

The laser is pulsed at a frequency of $1-3 \mathrm{~Hz}$. As the laser beam enters the chamber, it ionizes TMAE gas and forms a plasma. Laser intensity attenuation due to photon absorption is observed in the $\hat{z}$ direction. A neutral pressure of $40 \mathrm{mTorr}$ is found to be optimal to minimize density gradients in the $\hat{z}$ direction and yet provide sufficient plasma density to detect microwave reflections.

The microwave transmitter/receiver is located $50 \mathrm{~cm}$ from the plasma sheet. The diodes are biased with a $3-5 \mu \mathrm{W}$ of power from a separate microwave source operating at 8.5 GHz. This provides a small offset for each diode which provides greater sensitivity for measurement of plasma parameters.

As the microwave radiation is reflected from the plasma sheet, a voltage deflection is noted on the diodes. This voltage is recorded at $1 \mathrm{GS} / \mathrm{s}$ on the Tektronix TDS 350 oscilloscope and recorded using LabVIEW data acquisition software.

The normalized reflection coefficient versus time is plotted in Fig. 5. Background reflections account for $1 \%$ of the reflected radiation. The diodes respond in less than $10 \mathrm{~ns}$ after the laser pulse at $t=0$.

During the next $150 \mathrm{~ns}$, the density gradients created by photon absorption diminish, and the reflection increases until the reflection coefficient approaches $97 \%$ at $t=150 \mathrm{~ns}$. The reflection level remains at approximately this level for $100 \mathrm{~ns}$ before gradually decreasing until signal levels fall below the noise level of the system.

Examining the amplitude and phase of the plasma reflection coefficient (Figs. 5 and 6) shows a phase shift of $\Delta \phi$ $=1.0$ radians for the first $500 \mathrm{~ns}$ of reflection. This abrupt phase shift is a combination of the fast density decay, and the strong dependence of the phase on plasma density revealed in the theoretical curves of Fig. 6. As the plasma density decays below the critical density, the phase shift changes less abruptly. Langmuir probe data show $350 \mathrm{~ns}$ to be the time when the plasma is well below critical density, and density

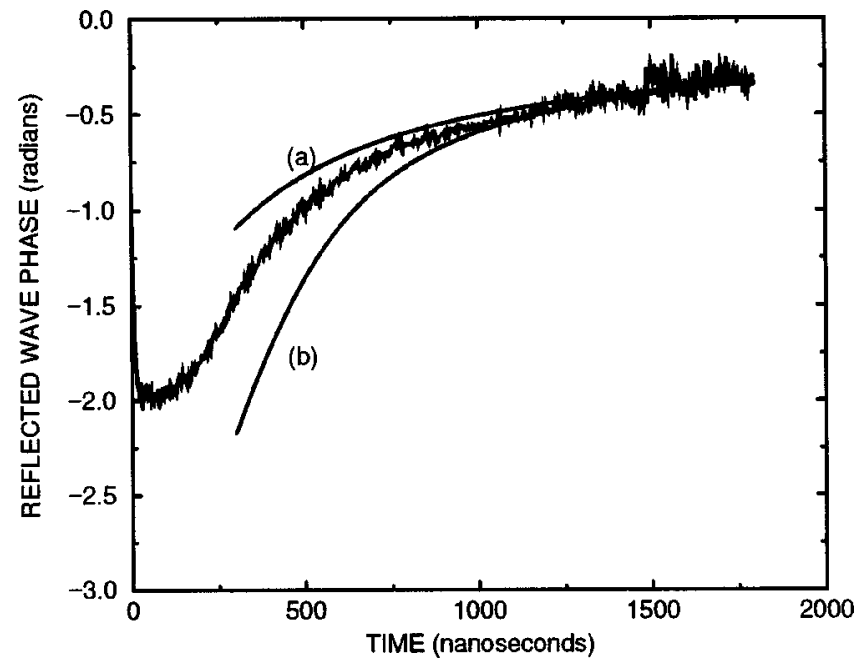

FIG. 6. Phase of reflected wave vs time for a $0.7 \mathrm{~cm}$ thick plasma (a) as simulated with a recombination coefficient $\alpha=2.0 \times 10^{-6} \mathrm{~cm}^{3} \mathrm{~s}^{-1}$, (b) $\alpha$ $=2.5 \times 10^{-6} \mathrm{~cm}^{3} \mathrm{~s}^{-1}$, (c) determined by actual measurement.

gradients have moderated to a point that plane waves can be assumed in the interior of the plasma sheet.

\section{COMPUTER SIMULATION}

A computer simulation of the experiment has been developed to interpret experimental data. In the simulation, a plane wave is introduced at the top vacuum-plasma interface and electric and magnetic field boundary conditions of both top and bottom plasma-dielectric interfaces are solved. The finite thickness of the plasma enhances the reflectivity when the plasma is below critical density due to the multiple boundary conditions. The simulation assumes a uniform density in the $\hat{y}$ direction. It takes into account two plasma dielectric interfaces about which reflections occur. The electric field reflection coefficient from the finite thickness plasma sheet is

$$
\Gamma^{\prime}=\frac{\eta_{p}^{\prime}-1}{\eta_{p}^{\prime}+1}
$$

where the input wave impedance $\eta_{p}^{\prime}$ is given by

$$
\eta_{p}^{\prime}=\eta \frac{1+\Gamma e^{2 i k d / \eta}}{1-\Gamma e^{2 i k d / \eta}}
$$

and the distance between the interfaces is $d$. The total phase change of the reflected wave over the duration of the experiment is a function of this distance. This thickness was obtained in the experiment by placing a $7 \mathrm{~mm}$ opaque aperture in front of the laser beam.

The simulated dielectric is composed of a plasma which decays in time according to Eq. (5). The simulations assume a negligible collision frequency which is reasonable for the frequency of interest. ${ }^{10}$

The recombination coefficient, $\alpha_{r}$, can be estimated by measuring the time rate of change of both the amplitude and phase of the reflected wave. The computer simulation was executed for several different values of $\alpha_{r}$. It was assumed that at $t=400 \mathrm{~ns}$ the plasma was in a source-free state and 
that Eq. (5) holds. Amplitude and phase data from the simulation were compared to amplitude and phase data from the experiment at $t=1800 \mathrm{~ns}$ and the simulation results were graphed along with what was observed in the laboratory. The value obtained by fitting the experimental amplitude and phase data to the amplitude and phase of the simulation is $\alpha_{r}=2.0( \pm 0.5) \times 10^{-6} \mathrm{~cm}^{3} \mathrm{~s}^{-1}$. This is in agreement with the value of $\alpha_{r}=2.0( \pm 0.3) \times 10^{-6} \mathrm{~cm}^{3} \mathrm{~s}^{-1}$ obtained by analyzing Langmuir probe data. Attention is given to the interpretation of reflection data at times $t>500 \mathrm{~ns}$ where plane waves propagate in the plasma. The results of the simulation in Figs. 5 and 6 show the dependence of the amplitude and phase of the reflection coefficient on $\alpha_{r}$. The laser energy for the experimental data in this case was $250 \mathrm{~mJ}$ corresponding to an energy flux of $40 \mathrm{~mJ} / \mathrm{cm}^{2}$.

\section{INTERPRETATION OF AXIAL DENSITY GRADIENT EFFECTS}

We can use Eq. (3) which is an expression for the reflection from an infinitely thick plasma as long as the wave is significantly attenuated within the plasma thickness. This will be the case for a plasma which is overdense.

The magnitude of the reflection coefficient from the sheet can be predicted by considering an exponential decrease in laser power due to photon absorption along the axial dimension of the laser beam which yields a variation in the plasma density

$$
n(z)=n_{0} e^{-\alpha_{z} z} .
$$

Such a variation is reasonable for times short in comparison with the initial recombination time $(t<50 \mathrm{~ns})$.

A plane wave impinging on the plasma surface is scattered with a phase which is dependent on the plasma density. This will have a noticeable effect on the received signal due to the reflected wave phase interference arising from axial density variations along the sheet.

To demonstrate the effect of this interference, we examine a collisionless plasma $(\nu=0)$ for which the reflection coefficient at normal incidence is given by

$$
\Gamma(z)=\frac{\eta_{p}(z)-1}{\eta_{p}(z)+1} .
$$

For a plasma which is overdense, $\eta_{p}$ is imaginary and the magnitude of the reflection from a differential section of the plasma is unity. The phase of this signal depends on the the plasma density according to

$$
\phi=-2 \tan ^{-1} \sqrt{\frac{n(z)}{n_{\text {crit }}}-1} .
$$

The reflected wave field is given by $\Gamma(z) E_{i}$ where $E_{i}$ is the incident wave field. Thus, $E_{r}$ has a $k_{z}$ spectrum which is given by

$$
E_{r}\left(k_{z}\right)=\int_{0}^{l} E_{i} \Gamma(z) e^{i k_{z} z} d z
$$

The $k_{z}$ spectrum causes the reflected wave to spread in $\hat{z}$ during its propagation back to the receiving horn. For a horn which is located at $y_{0}$ above the plasma, only the compo-

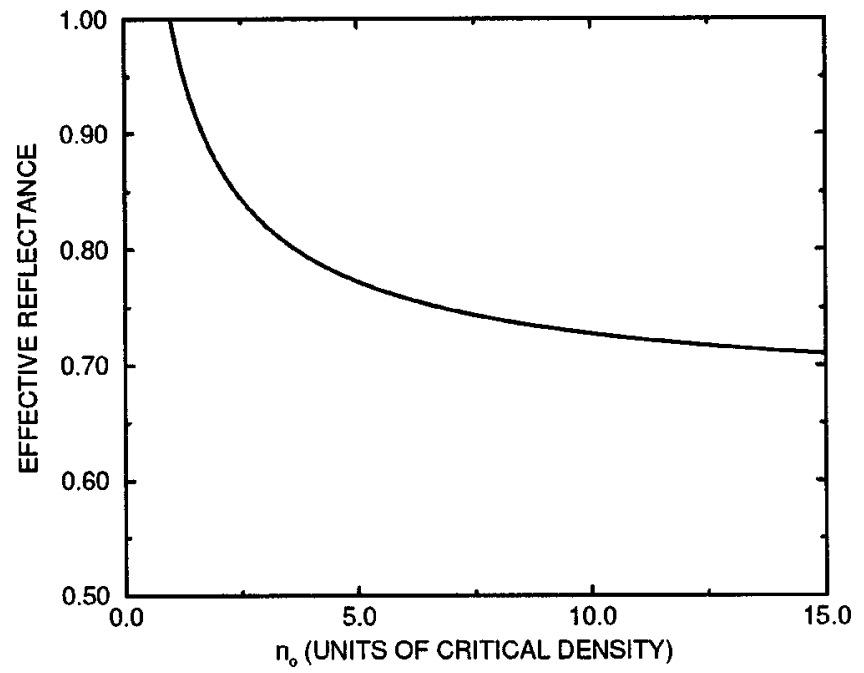

FIG. 7. Effective reflectance vs peak plasma density $n_{0}$ for exponentially varying plasma density.

nents in the spectrum with $\left|k_{z} / k_{0}\right|<l / 2 y_{0}$ will be received by the horn, the effective amplitude of the reflected wave is reduced to approximately

$$
E_{r} \approx E_{i} \frac{1}{l} \int_{0}^{l} \Gamma(z) d z
$$

The backscatter from the entire sheet with a length of $l$ is then

$$
\Gamma_{\text {sheet }}=\frac{1}{l} \int_{0}^{l} \frac{1-i \sqrt{n^{\prime}-1}}{1+i \sqrt{n^{\prime}-1}} d z,
$$

where $n^{\prime}$ denotes the normalized density $n(z) / n_{\text {crit }}$.

Assuming the density at $z=l$ to be the critical density, $\Gamma_{\text {sheet }}$ becomes

$$
\begin{aligned}
\Gamma_{\text {sheet }}= & \frac{2}{\ln \left(n_{0}^{\prime}\right)}\left[\frac{-1}{n_{0}^{\prime}}+1-\frac{1}{2} \ln \left(n_{0}^{\prime}\right)\right. \\
& \left.+i\left(\frac{\sqrt{n_{0}^{\prime}-1}}{n_{0}^{\prime}}+\tan ^{-1} \sqrt{n_{0}^{\prime}-1}\right)\right]
\end{aligned}
$$

and the received power is proportional to the effective reflectance $\left|\Gamma_{\text {sheet }}\right|^{2}$.

A graph of effective reflectance in Fig. 7 shows that for such an axial density gradient, the backscatter reflection coefficient of a plasma in which the density varies exponentially from $15 \times n_{\text {crit }}$ to $n_{\text {crit }}$ will be $70 \%$ of that for a uniform sheet. An interpretation of this graph is that a plasma sheet with a critical density at one end, and a density which varies exponentially up to $n_{0}$ at the other end will have an effective backscatter reflectance as shown by the graph. This reduction in received energy comes from the nonuniform density profile on the surface of the plasma and is produced by the narrow microwave beam being scattered into a broader angular pattern. One could view this as if the reflections come from a curved conducting sheet. Segments of the plasma which are sufficiently overdense experience a phase delay, and the effect of this phase delay on the reflected signal is similar to what one would observe if the conducting sheet 
was curved. This supports the observation of backscatter reflections of less than $100 \%$ for early times. As the density gradients are diminished due to the rapid recombination rate, the sheet's uniformity better characterizes a uniform conducting sheet and increased backscatter reflectivity is noted.

\section{SUMMARY}

A plasma sheet created by means of a VUV laser can be used to reflect substantial amounts of microwave radiation in the $X$-band range. The amount of radiation detected is a function of the plasma density as well as gradients in the plasma density. Phase interference caused by these gradients serves to broaden the beamwidth of the antenna used in this experiment.

Recombination rates are a determining factor in calculating the duration of reflections. The working gas in this experiment, TMAE, was found to have a recombination rate of $\alpha=2.0( \pm 0.5) \times 10^{-6} \mathrm{~cm}^{3} \mathrm{~s}^{-1}$. This figure is significantly smaller than the recombination rate for TMAE observed by Stalder et $a l .{ }^{4}$ where the recombination was studied with a helium background gas introduced. We found that the high laser energy $\left(E_{\text {laser }}=220-300 \mathrm{~mJ}\right)$ used for this experiment resulted in significantly longer plasma lifetimes than that observed by Stalder et al. where the VUV radiation was produced by spark gaps. Evidence of these longer lifetimes can be seen by noting significant microwave reflections from this plasma for times $t>500 \mathrm{~ns}$. A possible consequence of the high laser energy is an increase in the electron-neutral collision cross section where some of the neutral TMAE molecules are in an excited state for short time scales after the laser pulse. This increase would result in a higher collision frequency early in time, and possible further ionization of the excited states. If this is the case, we expect significant microwave absorption until the excited states decay. Evidence of this was noted as the reflectivity did not attain it's maximum value of $97 \%$ until nearly 300 ns after the laser was extinguished. Further studies of this absorption are needed to accurately determine collisionality and the existence of excited states. Microwave reflections lasting over $1.8 \mu$ s after the $20 \mathrm{~ns}$ laser pulse are observed with TMAE being ionized by means of a VUV excimer laser.

\section{ACKNOWLEDGMENTS}

This work is primarily supported by Air Force Office of Scientific research grants (Grant Nos. F49620-94-1-0054 and F49620-97-1-0262) and Air Force Office of Scientific Research Augmentation Award for Science and Engineering Training (Award No. F49620-93-1-0465) in cooperation with the Defense Department Research and Engineering Air Plasma Ramparts Multi-Disciplinary University Research Initiative program. It is also supported in part by the University of Wisconsin.

${ }^{1}$ R. A. Meger, J. Mathew, J. A. Gregor, R. E. Pechacek, R. F. Fernsler, W. Manheimer, and A. E. Robson, Phys. Plasmas 2, 2532 (1995).

${ }^{2}$ R. J. Vidmar, IEEE Trans. Plasma Sci. 18, 733 (1990).

${ }^{3}$ W. Manheimer, IEEE Trans. Plasma Sci. 19, 1228 (1991).

${ }^{4}$ K. R. Stalder, R. J. Vidmar, and D. J. Eckstrom, J. Appl. Phys. 72, 5089 (1992).

${ }^{5}$ J. E. Scharer, O. C. Eldridge, S. R. Chang, Y. S. Zhang, M. H. Bettenhausen, and N. T. Lam, IEEE Trans. Plasma Sci. 21, 271 (1993).

${ }^{6}$ J. D. Jackson, Classical Electrodynamics (Wiley, New York, 1975).

${ }^{7}$ W. Shen, J. E. Scharer, N. T. Lam, B. G. Porter, and K. L. Kelly, J. Appl. Phys. 78, 6974 (1995).

${ }^{8}$ C. A. Balanis, Antenna Theory-Analysis and Design (Wiley, New York, 1982).

${ }^{9}$ Y. Nakato, M. Ozaki, A Egawa, and H. Tsubomura, Chem. Phys. Lett. 9, 615 (1971).

${ }^{10}$ A. E. Robson, R. L. Morgan, and R. A. Meger, IEEE Trans. Plasma Sci. 20, 1036 (1992).

${ }^{11}$ D. F. Anderson, IEEE Trans. Nucl. Sci. NS-28, 842 (1981). 